\title{
Mechanisms Underlying Referral of Thermal Sensations to Sites of Tactile Stimulation
}

\author{
Hsin-Ni Ho (何昕霓), ${ }^{1}$ Junji Watanabe (渡邊淳司), ${ }^{1,2}$ Hideyuki Ando (安藤英由樹), ${ }^{3}$ and Makio Kashino (柏野牧夫) ${ }^{1,4}$ \\ ${ }^{1}$ NTT Communication Science Laboratories, NTT Corporation, Atsugi, Kanagawa 243-0198, Japan, ${ }^{2}$ Japan Society for the Promotion of Science, Atsugi, \\ Kanagawa 243-0198, Japan, ${ }^{3}$ Graduate School of Information Science and Technology, Osaka University, Suita, Osaka 565-0871, Japan, \\ and ${ }^{4}$ Interdisciplinary Graduate School of Science and Engineering, Tokyo Institute of Technology, Yokohama, Kanagawa 226-8503, Japan
}

When three stimulators are simultaneously touched with the middle three fingers of one hand but only the outer two stimulators are cooled or heated, the central (neutral) stimulator is also perceived to be cold or warm. This phenomenon is known as thermal referral and it shares phenomenological similarities with filling-in, in which the discontinuity in the signals of interest can be compensated perceptually on the basis of the spatially adjacent context. Although the mechanisms underlying filling-in have been well substantiated, those underlying thermal referral are still poorly understood. In the present study, we examined the intensity perception of the sensation resulting from thermal referral with human participants. We found that the sensation was uniform among the three fingers, but its apparent intensity was always lower than the physical intensity applied to the outer two fingers. These results indicate that the thermal uniformity perceived under thermal referral is not created by the brain's interpolating the thermal changes applied to the outer two fingers, as one would expect for those induced by typical filling-in. Instead, the thermal changes applied to the outer two fingers are summated and redistributed to all the fingers in contact. Our findings suggest that thermal referral is mediated by two separate processes. One determines the apparent intensity from the physical intensity and the areal extent of the thermal stimulation; the other determines the localization of the resulting sensation from the apparent sites of tactile stimulation.

\section{Introduction}

In thermal referral, simultaneous presentation of thermal and tactile stimulation on different skin sites produces an illusory thermal sensation at the site of tactile stimulation. This illusion was first demonstrated in an experiment where three stimulators were touched with the middle three fingers of one hand (from D2: index finger to D4: ring finger). When the outer two stimulators were cold (warm) and the center stimulator was thermally neutral, cold (warmth) was felt at all three fingers (Green, 1977). Thermal referral shares phenomenological similarities with filling-in, in which the discontinuity in the signals of interest can be compensated perceptually from the spatially or temporally adjacent context (for review, see Pessoa et al., 1998; Warren, 1999; Komatsu, 2006). One thing that distinguishes thermal referral from typical filling-in phenomena is the involvement of cross-modal processing. Concurrent tactile stimulation presented to D3 is essential for thermal referral, given that the illusory thermal sensation perceived at D3 disappears if D3 is held above the central (neutral) stimulator (Green, 1977). Thus, thermal referral can be regarded as a way to compensate for the discontinuity in the thermal characteristics of the sites in contact, so that a unified perceptual experience that is coherent across thermal and tactile modalities can be established.

\footnotetext{
Received May 20, 2010; revised 0ct. 20, 2010; accepted 0ct. 20, 2010.

Correspondence should be addressed to Hsin-Ni Ho, Human and information science laboratory, NTT Communication Science Laboratories, NTT Corporation, 3-1 Morinosato Wakamiya, Atsugi, Kanagawa, 243-0198, Japan. E-mail: hsinni@avg.brl.ntt.co.jp.

DOI:10.1523/JNEUROSCI.2640-10.2011

Copyright $\odot 2011$ the authors $\quad 0270-6474 / 11 / 310208-06 \$ 15.00 / 0$
}

Although the mechanisms underlying filling-in have been well substantiated, those underlying thermal referral are still poorly understood. It would be interesting to know whether or not the involvement of thermo-tactile interactions leads to a sensation that is different from those induced by typical filling-in. In case of typical filling-in, the filled-in information is in general a "copy" of the sensory inputs acquired in the surround, so that the resulting sensation is either spatially uniform, such as the sensations reported in the completion of color, luminance, and textures across a blind spot and scotomata (Ramachandran et al., 1993; Gyoba, 1997), or temporally continuous, such as the continuity illusions reported in auditory and vibrotactile stimulation (Warren, 1999; Kitagawa et al., 2009). To clarify whether the resulting sensation of thermal referral also has similar characteristics, we examined the uniformity of the sensation resulting from thermal referral and measured its apparent intensity as a function of the physical intensity applied to D2 and D4. We found that the resulting thermal sensation was perceived to be uniform among the three fingers, but the apparent intensity of the resulting sensation was always lower than the physical intensity applied to D2 and D4. Our findings indicate that the illusory thermal sensation perceived at D3 is not simply a "copy" of the thermal changes applied to D2 and D4, but instead results from perceptual summation and redistribution of the applied thermal changes to all the fingers in contact. On the basis of our findings, we suggest that thermal referral is mediated by two separate processes. One determines the apparent intensity of the resulting thermal sensation on the basis of the physical intensity and the areal extent of the thermal 
stimulation. The other redistributes the resulting thermal sensation to the apparent sites of tactile stimulation.

\section{Materials and Methods}

Participants. Four men and three women participated in experiments 1 and 2, and three men and four women participated in experiment 3 . Three participants were common to all three experiments. All the participants were right-handed, aged between 23 and 32 years, and had no known abnormalities of their tactile and thermal sensory systems. Recruitment of participants and experimental procedures were conducted in accordance with the Declaration of Helsinki.

Apparatus. Thermal displays that can present individually controlled thermal stimuli were used in the experiments. In experiment 1 (uniformity perception), one display was used to present thermal stimulation to the middle three fingers of one hand. In experiments 2 and 3 (intensity perception), two displays were used to present thermal stimulation to both hands. The thermal display consisted of three Peltier devices with a surface area of $20 \mathrm{~mm} \times 20 \mathrm{~mm}$ (FPH1-7106M, Fujitaka Co., Ltd.). A PI control loop programmed in VB.NET was used to control the surface temperatures of the Peltier devices. The maximum rate of temperature change was $10^{\circ} \mathrm{C} / \mathrm{s}$ for cooling and $18^{\circ} \mathrm{C} / \mathrm{s}$ for heating. Achieving a steady state took $\sim 1 \mathrm{~s}$. After a steady state has been reached, the temperature of each Peltier device can be maintained within $0.5^{\circ} \mathrm{C}$ of the desired temperature. The baseline temperature of both hands was controlled with a commercial hotplate (LS15P, Hakko).

Experiment 1: Uniformity perception. An A-Not-A procedure was adopted to examine the uniformity of the thermal sensation resulting from thermal referral. Thermal referral stimulation and physically uniform stimulation were presented to the middle three fingers of the right hand randomly and the participants were asked to report whether the thermal sensation perceived at the three fingers felt uniform. The experiment was conducted with the baseline temperature of the hand maintained at $32^{\circ} \mathrm{C}$. In thermal referral stimulation, only the outer two stimulators were cooled or heated. In physically uniform stimulation, all three stimulators were cooled or heated. The physical intensities, $\Delta T_{\mathrm{s}}$, applied were $-5,-7$, or $-10^{\circ} \mathrm{C}$ for cooling and 5,7 , or $10^{\circ} \mathrm{C}$ for warming (relative to the baseline temperature). The selected intensities were well above the thermal discrimination thresholds, which are $<1^{\circ} \mathrm{C}$ on the fingers and hand (Stevens and Choo, 1998), so it was expected that the participants would be able to detect the nonuniformity if the resulting thermal sensation did not feel spatially uniform. In addition, the selected intensities were all below the thermal pain threshold typically described (Hardy et al., 1952; Harrison and Davis, 1999). Given that it has been reported that in some individuals mild cooling and warming can also evoke painful sensations (Green, 2002; Green and Schoen, 2007), we tested whether any of our participants would have this low-threshold thermal nociception. At the beginning of the experiment, the participants experienced all the selected intensities and were asked to report whether or not the temperature stimulations felt painful. None of the participants reported painful sensations in any of the trials.

During the experiment, the cooling $\left(\Delta T_{\mathrm{s}}<0\right)$ and warming trials $\left(\Delta T_{\mathrm{s}}>0\right)$ were blocked. At the beginning of each block, the participants placed their hands on the hotplate to adapt their skin temperature to $32^{\circ} \mathrm{C}$. Each trial began with the participants placing their right hand on the hotplate. Upon hearing a sound cue, the participants moved their right hand from the hotplate and made contact with the stimulators for $5 \mathrm{~s}$. At the end of the contact, another sound cue was presented and the participants reported whether or not the thermal sensation perceived was uniform among the fingers in contact. The participants then returned their hands to the hotplate to await the start of the next trial. The intertrial interval was $\sim 20 \mathrm{~s}$. Within each block, three physical intensities $\left(\Delta T_{\mathrm{s}}=\right.$ $-5,-7,-10^{\circ} \mathrm{C}$ for cooling; $\Delta T_{s}=5,7$, or $10^{\circ} \mathrm{C}$ for warming) were repeated seven times for both thermal referral stimulation and physically uniform stimulation to give a total of 42 trials $(3 \times 7 \times 2$ trials). These trials were presented in a randomized order and therefore the thermal intensity perceived on the fingers was different from trial to trial. The participants were instructed to focus on the uniformity of the sensation and respond regardless of the intensity perceived.
Experiment 2: Intensity perception under thermal referral. The adaptive staircase method was used to measure the apparent intensity of the thermal sensation resulting from thermal referral as a function of the physical intensity applied to the outer two fingers. The baseline temperature and the physical intensities used were the same as those in experiment $1\left(32^{\circ} \mathrm{C}\right.$ for baseline temperature; $\Delta T_{\mathrm{s}}=-5,-7$, or $-10^{\circ} \mathrm{C}$ for cooling and 5,7 , or $10^{\circ} \mathrm{C}$ for warming). The middle three fingers of the right hand were presented with the thermal referral stimulation with a fixed intensity, $\Delta T_{\mathrm{s}}$, while the corresponding fingers of the left hand were presented with the physically uniform stimulation with intensity, $\Delta T_{\mathrm{u}}$, varied adaptively. The participants compared the overall thermal intensity of one hand to that of the other. For cooling trials, $\Delta T_{\mathrm{u}}$ was decreased when a participant responded that the right hand felt colder and was increased when the participant responded that it felt warmer. Similarly, for warming trials, $\Delta T_{\mathrm{u}}$ was increased when a participant responded that the right hand felt warmer and was decreased when the participant responded that it felt colder. For each $\Delta T_{\mathrm{s}}$, the staircase started randomly with $\Delta T_{\mathrm{u}}$ in the range of $\left(\Delta T_{\mathrm{s}}-3\right)$ and $\left(\Delta T_{\mathrm{s}}+8\right)^{\circ} \mathrm{C}$ for cooling trials and $\left(\Delta T_{\mathrm{s}}-8\right)$ and $\left(\Delta T_{\mathrm{s}}+3\right)^{\circ} \mathrm{C}$ for warming trials, so that the initial $\Delta T_{\mathrm{u}}$ would not exceed the thermal pain threshold typically described. Each staircase included 15 trials. The initial step size was $3^{\circ} \mathrm{C}$, and after the first reverse point, the step size was decreased to $2^{\circ} \mathrm{C}$. The step size was set at 1 and $0.5^{\circ} \mathrm{C}$, respectively after the third and fifth reversal points. To prevent painful sensations and skin damage, the maximum $\Delta T_{\mathrm{u}}$ provided throughout the staircase never exceeded $-15^{\circ} \mathrm{C}$ for cooling trials and $13^{\circ} \mathrm{C}$ for warming trials.

Each participant came to the lab for $2 \mathrm{~d}$ and was tested with the cooling trials on $1 \mathrm{~d}$ and the warming trials on the other. The participants' skin temperature was maintained at $32^{\circ} \mathrm{C}$ at the beginning of each staircase and each trial of the staircase began with the participants placing their hands on the hotplate. Upon hearing a sound cue, the participants moved both of their hands from the hotplate and made contact with the stimulators for $5 \mathrm{~s}$. At the end of the contact, another sound cue was presented and the participants reported whether the right hand felt cooler (warmer) than the left hand. The participants then returned their hands to the hotplate to await the start of the next trial. The intertrial interval was $\sim 20 \mathrm{~s}$. For each thermal condition, three staircases were conducted for each intensity level to give a total of nine staircases. These staircases were presented in a randomized order.

Experiment 3: Intensity perception when D3 was not in contact. The experimental procedure for experiment 3 were the same as that in experiment 2 except that the participants held D3 above the central (neutral) stimulator to eliminate the tactile stimulation. Under this condition, no referral occurred (Green, 1977). Thermal neutrality was therefore perceived on D3, while cold/warmth was perceived on the outer two fingers. In the experiment, D2 and D4 of the right hand were presented with intensity, $\Delta T_{\mathrm{s}}$, while D2, D3 and D4 of the left hand were presented with the physically uniform stimulation with intensity, $\Delta T_{\mathrm{u}}$, varied adaptively. The participants compared the overall thermal intensity of one hand to that of the other.

Statistical analyses. In experiment 1 (uniformity perception), a signal-detection-based analysis was performed on the responses to the experimental trials. A hit was defined as a "not uniform" response when the thermal referral stimulation was presented, while false alarm was defined as a "not uniform" response when physically uniform stimulation was presented. Sensory discriminability, $d^{\prime}$, defined as $z\left(P_{\mathrm{Hit}}\right)-z\left(P_{\mathrm{FA}}\right)$, was then estimated from the hit rate and false alarm rate for sensory discrimination between thermal referral stimulation and physically uniform stimulation. In experiments 2 and 3 (intensity perception), the average of the last four reversal points of each staircase was used to determine the apparent intensity of the corresponding resulting thermal sensation, $\Delta T_{\mathrm{a}}$. For cooling trials, the absolute values of the physical and apparent intensities were used in the statistical analyses. The significance level of the statistical analyses was set at 0.05 . All analyses were performed with SPSS and the Matlab statistics toolbox. 


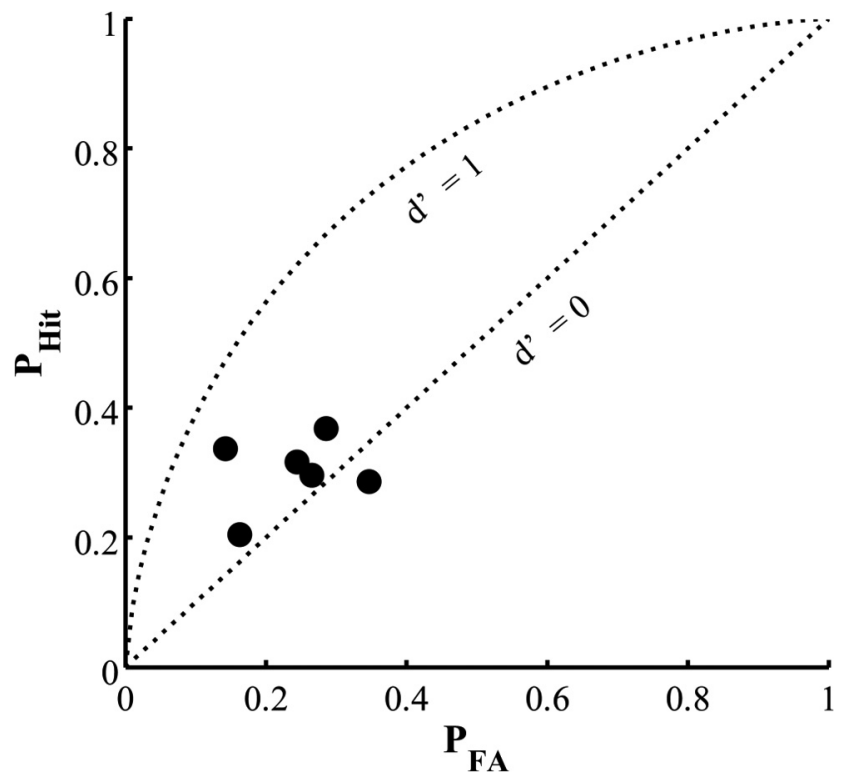

Figure 1. Hit rate versus false alarm rate for discriminating the thermal referral simulation from the physically uniform stimulation on the basis of the apparent uniformity. Each point represents the group mean performance for a physical intensity. The curves represent the locations corresponding to $d^{\prime}=0$ and 1 .

\section{Results}

\section{Experiment 1: Uniformity perception}

The results showed that the participants were not able to discriminate the thermal referral stimulation from the physically uniform stimulation reliably on the basis of their apparent uniformity. Figure 1 plots the group mean hit rate versus the group mean false alarm rate for each physical intensity tested. All the points are clustered between the curves of $d^{\prime}=0$ and $d^{\prime}=1$, indicating that the participants were not able to discriminate between the uniformity induced by thermal referral stimulation and the physically uniform stimulation. This lack of discriminability was also indicated by an adjusted Pearson's $\chi^{2}$ test with Yate's continuity correction ( $\mathrm{Bi}, 2006)$, which confirmed that there was no significant difference in the apparent uniformity induced by the thermal referral simulation and the physically uniform stimulation for all intensities tested $(p>0.05$ for all intensities). These results indicate that the resulting sensation of thermal referral is spatially uniform and indistinguishable from that induced by the physically uniform stimulation.

\section{Experiment 2: Intensity perception under thermal referral}

In this experiment, we measured the apparent intensity of the resulting sensation of thermal referral, $\Delta T_{\mathrm{a}}$, as a function of the physical intensity applied to D2 and D4, $\Delta T_{\mathrm{s}}$, which were $-5,-7$, or $-10^{\circ} \mathrm{C}$ for cooling and 5,7 , or $10^{\circ} \mathrm{C}$ for warming (relative to the baseline temperature). Because the resulting sensation was shown to be spatially uniform in experiment 1 , we would like to know whether its apparent intensity is the same as that induced by applying physically uniform stimulation to all three fingers.

The results are shown in Figure 2. The line of equality in Figure 2 represents the results expected when the apparent intensity of the resulting sensation is exactly the same as that induced by the physically uniform stimulation. If the data points were on this line, it would mean that the thermal sensation perceived at D3 was a "copy" of the thermal changes applied to D2 and D4. However, our results show that the apparent intensity of the resulting sensation was significantly lower than the physical inten-

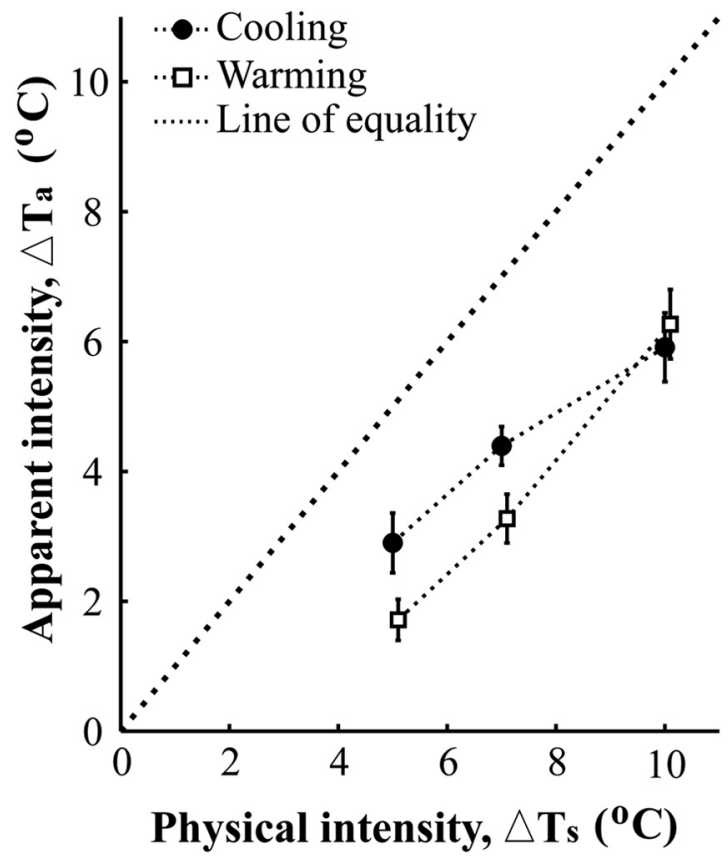

Figure 2. Group mean of the apparent intensity under thermal referral as a function of the physical intensity for cooling (filled circles) and warming (open square). Error bars show the SEM. Note that apparent and physical intensities are plotted in absolute values, so the intensities under cooling conditions are also positive.

sity applied to D2 and D4 for both cooling and warming conditions, as indicated by one-sample $t$ tests that compared apparent intensity against the corresponding physical intensity for all the intensities tested ( $p<0.01$ for all intensities). Our results indicate that although the resulting sensation of thermal referral was perceived to be uniform among D2, D3, and D4, this uniformity was not created by the brain's interpolating the thermal changes applied to D2 and D4. Instead, the apparent intensity on D2 and D4 decreased from $\Delta T_{\mathrm{s}}$ to $\Delta T_{\mathrm{a}}$ and the apparent intensity on D3 increased toward $\Delta T_{\mathrm{a}}$ as illustrated in Figure 3. As a result, a uniform sensation with lower apparent intensity is perceived.

A repeated-measure ANOVA was performed with physical intensity and thermal conditions (cooling/warming) as withinsubject factors and apparent intensity as the dependent variable. The analysis indicated that the physical intensity has a significant effect on the apparent intensity $\left(F_{(2,12)}=108.54, p<0.001\right)$, while there was no main effect of thermal condition $\left(F_{(1,6)}=0.78\right.$, $p=0.41)$. A significant two-way interaction was found between the effects of physical intensity and thermal condition $\left(F_{(2,12)}=\right.$ $4.14, p=0.04)$. To understand the nature of this interaction, we tested for simple main effects between cooling and warming under each physical intensity. The difference between cooling and warming was shown to be significant at $5^{\circ} \mathrm{C}$ $\left(F_{(1,12)}=7.65, p=0.02\right)$ and $7^{\circ} \mathrm{C}\left(F_{(1,12)}=6.82, p=0.02\right)$. These results suggest that the intensity perception under cold and warm referrals is different.

\section{Experiment 3: Intensity perception when D3 was not in contact}

The results of experiments 1 and 2 indicate that the resulting thermal sensation of thermal referral was perceived to be uniform among the fingers (experiment 1), but its apparent intensity was always lower than the physical intensity applied to the outer two fingers (experiment 2). These findings suggest that the illusory thermal sensation perceived at D3 results from perceptual sum- 


\section{Thermal referral stimulation}

a Warming $\left(\Delta T_{S}>0\right)$

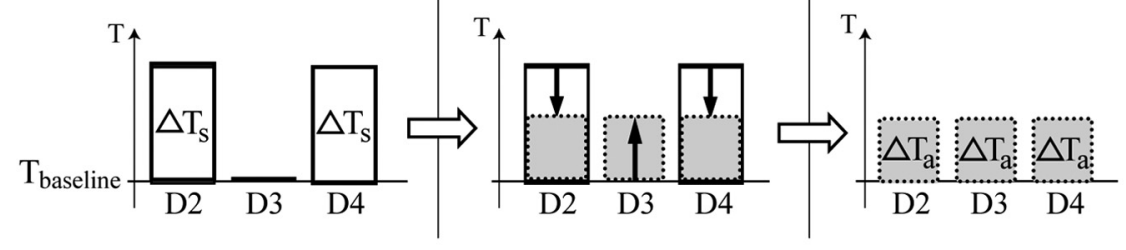

b Cooling $\left(\Delta \mathrm{T}_{\mathrm{S}}<0\right)$

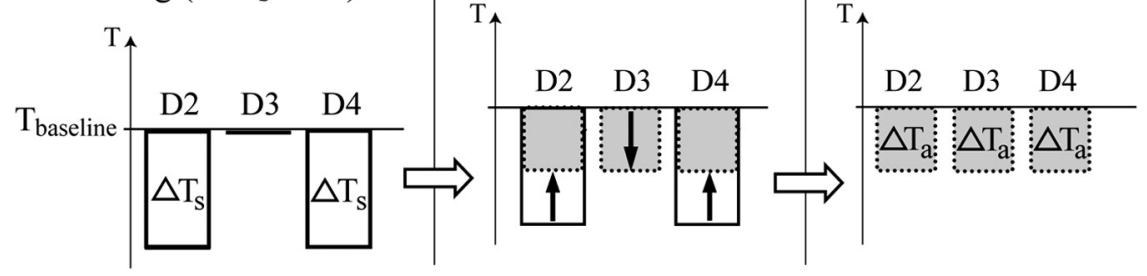

Figure 3. Diagrams illustrating what happened under thermal referral in warming condition $(\boldsymbol{a})$ and cooling condition (b). The leftmost panel represents the physical stimulation applied and the rightmost panel shows the resulting thermal sensation. The physical intensity $\left(\Delta T_{\mathrm{s}}\right)$ and the apparent intensity $\left(\Delta T_{\mathrm{a}}\right)$ are the changes in temperature applied and perceived with respect to the baseline temperature.

mation and redistribution of the applied thermal changes to all the fingers in contact. To test this summation theory, we investigated the intensity perception when D3 was not in contact in experiment 3. Our logic is that if the summation theory is true, the overall intensity perceived will not vary with $\mathrm{D} 3$ contact, since the physical intensity and the areal extent of the thermal stimulation were the same in both cases $\left(\Delta T_{\mathrm{s}}\right.$ to D2 and D4). In the experiment, D2 and D4 of the right hand were presented with intensity, $\Delta T_{\mathrm{s}}$, while D2, D3 and D4 of the left hand were presented with the physically uniform stimulation. The apparent intensity, $\Delta T_{\mathrm{a}}$, was defined as the intensity applied to the physically uniform stimulation when the overall intensity perceived at both hands was comparable.

The results are shown in supplemental Figure 1 (available at www.jneurosci.org as supplemental material). The line of equality represents the results expected when the apparent intensity, $\Delta T_{\mathrm{a}}$ is equal to the physical intensity $\Delta T_{\mathrm{s}}$, applied to D2 and D4. The apparent intensity, $\Delta T_{\text {a }}$, was always lower than line of equality, indicating a trade-off between the physical intensity and areal extent of thermal stimulation $\left(\Delta T_{\mathrm{s}}\right.$ to two fingers versus $\Delta T_{\mathrm{a}}$ to three fingers). In addition, the apparent intensity in the contact condition was in general lower than that in the no-contact condition, which is consistent with the finding that active touch can attenuate the thermal sensations on the hand (Green, 2009).

Separate ANOVAs with whether D3 was in contact as the between-subject factor and the apparent intensity $\Delta T_{\mathrm{a}}$ from experiments 2 and 3 as the dependent variable were conducted for cooling and warming conditions. The analyses indicated that the apparent intensity did not change significantly under the cooling condition ( $p=0.54$ ) and fell just short of significance under the warming condition $(p=0.052)$. Although the marginally significant results of warming condition might suggest that, in addition to the physical intensity and the areal extent of the thermal stimulation, other factors, such as the tactile contacts, could also exert an influence, the results in general support our theory that the overall intensity perceived would not vary with D3 contact.

\section{Discussion}

\section{Principal findings}

The resulting thermal sensation of thermal referral was perceived to be uniform among the fingers (experiment 1), but its apparent intensity was always lower than the physical intensity applied to the outer two fingers (experiment 2). Our findings indicate that the thermal uniformity perceived under thermal referral is in fact not created by the brain's interpolating the thermal changes applied to the outer two fingers, as one would expect for those induced by typical perceptual filling-in. Instead, the thermal changes applied to D2 and $\mathrm{D} 4$ are summated and redistributed to all the fingers in contact so that a uniform sensation with lower apparent intensity is perceived (Fig. 3).

Our findings suggest that thermal referral is mediated by two separate processes. One process determines the overall intensity of the resulting sensation from the physical intensity and the areal extent of the thermal stimulation. This process is known as spatial summation in the literature of thermal perception and has been studied extensively in various body regions, including the forehead, forearm, and back (Kenshalo et al., 1967; Stevens and Marks, 1971, 1979). To test our theory that the overall intensity is determined from the physical intensity $\left(\Delta T_{\mathrm{s}}\right)$ and the areal extent of the thermal stimulation (D2 and D4), we investigated the intensity perception when $\mathrm{D} 3$ was not in contact in experiment 3. The results indicated that the overall intensity did not vary with D3 contact, which supports our theory.

The second process redistributes the overall thermal intensity determined in the first process to the apparent sites of the tactile stimulation (D2, D3, and D4), so that a uniform thermal sensation is perceived among these three fingers with a lower apparent intensity. This "mislocalization" of the thermal sensations presumably results from the tactile dominance over the thermal modality in localization, as the spatial acuity of the tactile modality has been shown to be superior to that of the thermal modality (Simmel and Shapiro, 1969; Cain, 1973). This may explain the absence of any thermal referral when D3 is not in contact and the referral of thermal sensations to the sites that are not thermally neutral (Green, 1977).

Although our findings are obtained with uniform temperature changes in the surround, like those used when Green originally discovered thermal referral (Green, 1977), it is also possible that thermal referral could occur when the changes in temperature in the surround are not of the same magnitude or direction (i.e., when warming and cooling are applied to each of the outer two fingers). Further investigations are needed to test our theory under those conditions.

\section{Illusory thermal uniformity}

Our results showed that the resulting sensation of thermal referral is spatially uniform and indistinguishable from that induced by the physically uniform stimulation. It is important to note that this illusory thermal uniformity has a spatial limit, which is dictated by the distance between and among the fingers being defined in the cortical topography (Green, 1978; Ho et al., 2010). 
When the distance among the fingers was increased by having D2 and D5 as the outer fingers and D3 as the neutral inner finger, the illusory thermal sensation could still be perceived on D3 but the intensity was reduced, so that the resulting sensation was not uniform across the three fingers. Increasing the distance further by replacing the ipsilateral D3 with the contralateral D3 would diminish the effect completely. Thus, the thermo-tactile interactions underlying thermal referral might reside in early cortical areas whose organization conserves the topographic information.

\section{Difference between cooling and warming under thermal referral}

Although cooling and warming stimulation of similar physical intensity normally arouses cooling and warming sensations with similar apparent intensity (Greenspan et al., 2003), our results indicate that there is a difference in intensity perception between referrals of cold and warm sensations. It is possible that the difference between cold and warm referrals reflects a fundamental difference between our senses of cold and warmth. Our sense of warmth has been shown to be more diffuse than our sense of cold in a spatial discrimination task (Hensel, 1982; Lee et al., 1996), and the spatial summation is greater for warming stimuli than for cooling stimuli (Greenspan and Kenshalo, 1985). It is also known that cold receptors are always more numerous than warm receptors in different body regions (Darian-Smith, 1984). These factors all could contribute to the difference in the referral of cold and warm sensations.

\section{Thermal referral and filling-in phenomenon}

One might doubt whether it is appropriate to regard thermal referral as a filling-in phenomenon. Although filling-in primarily refers to the completion of missing information across a physiological blind spot and across natural and artificial scotomata (Ramachandran and Gregory, 1991; Ramachandran, 1992), in a more general definition, it refers to situations in which observers report that something is present in a particular region even though it exists only in the surround. According to this definition, visual illusions, such as neon color spreading (Bressan et al., 1997) and the Craik-O'Brien-Cornsweet illusion (Cornsweet, 1970; Dooley and Greenfield, 1977) and tactile illusions, such as the cutaneous rabbit (Geldard and Sherrick, 1972) have also been discussed in terms of filling-in (Pessoa et al., 1998; Komatsu, 2006; Kitagawa et al., 2009). Given that thermal referral shares phenomenological similarities with filling-in, we believe it is appropriate to make comparisons between thermal referral and filling-in.

There are two major theories about the underlying mechanisms of filling-in. One is known as the symbolic or cognitive theory, which argues that the missing information is reconstructed in higher cortical areas, so there is no need for neuronal activity to occur in the region where filling-in is perceived. The other is known as the isomorphic theory, which assumes that when filling-in occurs, neuronal activity propagates in the early cortical areas that still preserve retinotopic information (Pessoa et al., 1998; Komatsu, 2006). As suggested by the isomorphic theory, a recent study of the tactile illusion, known as the cutaneous rabbit illusion, demonstrated that neuronal activity is elicited at a somatotopic location corresponding to the filled-in illusory perception in primary somatosensory cortex (Blankenburg et al., 2006). Although it is suggested that thermal referral also involves neuronal activities in early cortical areas (Ho et al., 2010), the neural computation involved in summation and redistribution of thermal sensations is expected to be more complicated than what is assumed in the isomorphic theory (see also a recent report on feature averaging in visual filling-in by Hsieh and Tse, 2009).

Although the neural mechanisms underlying thermal referral and filling-in might be distinct, the functional significance of both is related to object recognition. Thermal referral resolves the spatial discrepancy between the thermal and tactile inputs, so that thermal and tactile information can be combined optimally to give a unified percept of the object to assist in object recognition (Lederman and Klatzky, 1997; Ho and Jones, 2006). It is thus important to understand the mechanism underlying thermal referral to deepen our knowledge about information processing during manual exploration, which is essential for acquiring information from the external world.

\section{References}

Bi J (2006) Sensory discrimination tests and measurements. Iowa: Blackwell Publishing Professional.

Blankenburg F, Ruff CC, Deichmann R, Rees G, Driver J (2006) The cutaneous rabbit illusion affects human primary sensory cortex somatotopically. PLoS Biol 4:459-466.

Bressan P, Mingolla E, Spillmann L, Watanabe T (1997) Neon color spreading: a review. Perception 26:1353-1366.

Cain WS (1973) Spatial discrimination of cutaneous warmth. Am J Psychol 86:169-181.

Cornsweet T (1970) Visual perception. New York: Academic.

Darian-Smith I (1984) Thermal sensibility. In: Handbook of physiology: the nervous system (Darian-Smith I, ed), pp 879-913. Bethesda, MD: American Physiological Society.

Dooley RP, Greenfield MI (1977) Measurements of edge-induced visual contrast and a spatial-frequency interaction of the Cornsweet illusion. J Opt Soc Am 67:761-763.

Geldard FA, Sherrick CE (1972) The cutaneous "rabbit": a perceptual illusion. Science 178:178-179.

Green BG (1977) Localization of thermal sensation: an illusion and synthetic heat. Percept Psychophys 22:331-337.

Green BG (1978) Referred thermal sensations: warmth versus cold. Sens Processes 2:220-230.

Green BG (2002) Synthetic heat at mild temperatures. Somatosens Motor Res 19:130-138.

Green BG (2009) Temperature perception on the hand during static versus dynamic contact with a surface. Atten Percept Psychophys 71:1185-1196.

Green BG, Schoen KL (2007) Thermal and nociceptive sensations from menthol and their suppression by dynamic contact. Behav Brain Res 176:284-291.

Greenspan JD, Kenshalo DR Sr (1985) The primate as a model for the human temperature-sensing system: 2 . area of skin receiving thermal stimulation. Somatosens Res 2:315-324.

Greenspan JD, Roy EA, Caldwell PA, Farooq NS (2003) Thermosensory intensity and affect throughout the perceptible range. Somatosens Motor Res 20:19-26.

Gyoba J (1997) Loss of a forest: Perceptual fading and filling-in of static texture patterns. Perception 26:1317-1320.

Hardy JD, Wolff HG, Goodell H (1952) Pricking pain threshold in different body areas. Proc Soc Exp Biol Med 80:425-427.

Harrison JL, Davis KD (1999) Cold-evoked pain varies with skin type and cooling rate: a psychophysical study in humans. Pain 83:123-135.

Hensel H (1982) Thermal sensations and thermoreceptors in man. Springfield, IL: Thomas.

Ho HN, Jones LA (2006) Contribution of thermal cues to material discrimination and localization. Percept Psychophys 68:118-128.

Ho HN, Watanabe J, Ando H, Kashino M (2010) Somatotopic or spatiotopic? Frame of reference for localizing thermal sensations under thermo-tactile interactions. Atten Percept Psychophys 72:1666-1675.

Hsieh PJ, Tse PU (2009) Feature mixing rather than feature replacement during perceptual filling-in. Vision Res 49:439-450.

Kenshalo DR, Decker T, Hamilton A (1967) Comparisons of spatial summation on the forehead, forearm, and back produced by radiant and conducted heat. J Comp Physiol Psychol 63:510-515. 
Kitagawa N, Igarashi Y, Kashino M (2009) The tactile continuity illusion. J Exp Psychol Hum Percept Perform 35:1784-1790.

Komatsu H (2006) The neural mechanisms of perceptual filling-in. Nat Rev Neurosci 7:220-231.

Lederman SJ, Klatzky RL (1997) Relative availability of surface and object properties during early haptic processing. J Exp Psychol Hum Percept Perform 23:1680-1707.

Lee DK, McGillis SLB, Greenspan JD (1996) Somatotopic localization of thermal stimuli: I. A comparison of within-versus across-dermatomal separation of innocuous thermal stimuli. Somatosens Motor Res 13:67-71.

Pessoa L, Thompson E, Noë A (1998) Finding out about filling-in: a guide to perceptual completion for visual science and the philosophy of perception. Behav Brain Sci 21:723-802.

Ramachandran VS (1992) Blind spots. Sci Am 266:86-91.
Ramachandran VS, Gregory RL (1991) Perceptual filling in of artificially induced scotomas in human vision. Nature 350:699-702.

Ramachandran VS, Gregory RL, Aiken W (1993) Perceptual fading of visual texture borders. Vision Res 33:717-721.

Simmel ML, Shaprio A (1969) The localization of non-tactile thermal sensations. Psychophysiology 5:415-425.

Stevens JC, Choo KC (1998) Temperature sensitivity of the body surface over the life span. Somatosens Motor Res 15:13-28.

Stevens JC, Marks LE (1971) Spatial summation and the dynamics of warmth sensation. Percept Psychophys 9:391-398.

Stevens JC, Marks LE (1979) Spatial summation of cold. Physiol Behav 22:541-547.

Warren RM (1999) Perceptual restoration of missing sounds. In: Auditory perception: a new analysis and synthesis, pp 134-154. Cambridge, UK: Cambridge UP. 